\title{
Nasal Vestibule
}

National Cancer Institute

\section{Source}

National Cancer Institute. Nasal Vestibule. NCI Thesaurus. Code C160989.

The most anterior part of the nasal cavity, lined by squamous epithelium. 